Segmentation-free statistical image reconstruction for polyenergetic $\mathrm{x}$-ray computed tomography with experimental validation

This article has been downloaded from IOPscience. Please scroll down to see the full text article.

2003 Phys. Med. Biol. 482453

(http://iopscience.iop.org/0031-9155/48/15/314)

View the table of contents for this issue, or go to the journal homepage for more

Download details:

IP Address: 141.213.32.90

The article was downloaded on 25/07/2011 at 16:21

Please note that terms and conditions apply. 


\title{
Segmentation-free statistical image reconstruction for polyenergetic $x$-ray computed tomography with experimental validation
}

\author{
Idris A Elbakri ${ }^{1}$ and Jeffrey A Fessler \\ Electrical Engineering and Computer Science Department, University of Michigan, \\ 1301 Beal Ave, Ann Arbor, MI 48109, USA \\ E-mail: ielbakri@fischerimaging.com and fessler@umich.edu
}

Received 17 March 2003

Published 22 July 2003

Online at stacks.iop.org/PMB/48/2453

\begin{abstract}
This paper describes a statistical image reconstruction method for x-ray CT that is based on a physical model that accounts for the polyenergetic $\mathrm{x}$-ray source spectrum and the measurement nonlinearities caused by energy-dependent attenuation. Unlike our earlier work, the proposed algorithm does not require pre-segmentation of the object into the various tissue classes (e.g., bone and soft tissue) and allows mixed pixels. The attenuation coefficient of each voxel is modelled as the product of its unknown density and a weighted sum of energydependent mass attenuation coefficients. We formulate a penalized-likelihood function for this polyenergetic model and develop an iterative algorithm for estimating the unknown density of each voxel. Applying this method to simulated x-ray CT measurements of objects containing both bone and soft tissue yields images with significantly reduced beam hardening artefacts relative to conventional beam hardening correction methods. We also apply the method to real data acquired from a phantom containing various concentrations of potassium phosphate solution. The algorithm reconstructs an image with accurate density values for the different concentrations, demonstrating its potential for quantitative CT applications.
\end{abstract}

\section{Introduction}

Recently, there has been a surge of interest in applying statistical iterative reconstruction techniques to x-ray computed tomography (De Man et al 2001, Elbakri and Fessler 2002, Sukovic and Clinthorne 2000, Williamson et al 2002). Unlike the conventional filtered back projection (FBP) method, iterative methods are based on models for the measurement statistics

1 Currently with Fischer Imaging Corporation. 
and physics, and have the potential for improved bias-variance performance. Iterative methods are also attractive because they are better suited for irregular (non-Radon) geometries and situations with truncated data.

Statistical reconstruction methods can be degraded by model mismatches. In addition to measurement statistics, accurate iterative algorithms should account for phenomena such as beam hardening, scatter, partial-volume effects, etc (De Man et al 2001).

This paper focuses on beam hardening effects (Brooks and Chiro 1976, Joseph and Spital 1978, Kak and Slaney 1988). Beam hardening is a process whereby the average energy of the $\mathrm{X}$-ray beam increases as the beam propagates through a material since lower energy $\mathrm{X}$-rays are preferentially attenuated. X-rays traversing different paths through an object will emerge with different spectra, leading to inconsistencies in the data (in the Radon sense) and reconstruction artefacts if one ignores the energy dependence of the measurements.

If uncorrected, beam hardening generally leads to a reduction in the reconstructed attenuation coefficient (Brooks and Chiro 1976). Thick bones also generate dark streaks (Kak and Slaney 1988). In soft tissue, non-uniform depression of image values, or 'cupping', results. In addition, bone areas can 'spill over' into soft tissue, leading to a perceived increase in the attenuation coefficient (Joseph and Spital 1978).

Beam hardening artefacts and correction schemes have been investigated since the early days of clinical x-ray CT. They arise in clinical practice because the x-ray attenuation properties of human tissue are energy-dependent, and x-ray sources in typical clinical scanners emit broad spectra. Monoenergetic photon sources would eliminate beam hardening artefacts but are impractical for diagnostic CT because of SNR considerations. Beam hardening correction methods are therefore necessary for reconstructing artifact-free attenuation coefficient images from polyenergetic measurements. There are a variety of schemes for eliminating beam hardening artefacts in FBP images, including dual-energy imaging and post-processing techniques (Alvarez and Macovski 1976, Brooks and Chiro 1976, Hsieh et al 2000, Joseph and Spital 1978, Meagher et al 1990, Nalcioglu and Lou 1979).

The dual-energy approach (Alvarez and Macovski 1976, Fessler et al 2002, Sukovic and Clinthorne 2000) doubles the number of measurements and enables reconstruction of separate bone and soft tissue images. It is useful for tissue characterization and quantitative CT, but its major drawback is the requirement for two separate energy measurements.

The method described by Joseph and Spital (henceforth referred to as JS) (Joseph and Ruth 1997, Joseph and Spital 1978) is a post-processing technique that corrects for soft tissue and dense bone distortions. The method involves an initial FBP reconstruction and segmentation of the image into soft tissue and bone images. The segmented images are forward projected. The bone projection provides an estimate of the amount of nonlinear beam hardening distortion, which is then corrected for. Applying FBP to the corrected data provides an image with reduced artefacts. It is also possible to iterate the procedure several times to improve the resulting image.

Yan et al (2000) developed an iterative, but non-statistical, beam hardening correction method. It also assumes two categories of materials and iteratively computes their volume fraction at each pixel.

Recently, De Man et al (2001) proposed an alternative statistical approach that models the object attenuation as a linear combination of the attenuation properties of two base substances. The algorithm uses the polyenergetic source spectrum and does not need a pre-segmented image. The algorithm can reconstruct mixed pixels but is not guaranteed to monotonically increase the likelihood each iteration.

In this paper, we present and demonstrate the effectiveness of a statistical reconstruction algorithm for X-ray CT. In our earlier work we presented a statistical algorithm that, like the JS 
method, required pre-segmenting an initial image and did not permit pixels to contain tissue mixtures (Elbakri and Fessler 2002). In the method we propose herein, no segmentation is necessary and pixels may contain mixtures. We present two possible parametric object models. The displacement object model is designed for objects consisting of two material components (e.g., bone and soft tissue) that are spatially distinct, with mixed pixels arising at material boundaries. The solution object model, on the other hand, is designed for imaging objects that consist of mineral solutions, such as the interior of bones, and maybe useful for bone densitometry and other quantitative applications. Both models are physically intuitive.

Prior knowledge of the X-ray spectrum may be used in the forward model, but is not necessary. Tabulated measurements of the line integrals of bone and water over a reasonable range of object thicknesses for the CT system of interest are sufficient. The algorithm iteratively minimizes surrogate functions to the Poisson likelihood. Although one version of the algorithm is monotonic, we usually use ordered subsets to accelerate the algorithm, which compromises monotonicity. The algorithm can also easily accommodate scatter estimates.

The paper is organized as follows. The next section discusses polyenergetic X-ray CT and derives the iterative algorithm. In section 3 we present simulation results that compare JScorrected FBP with the proposed statistical reconstruction algorithm. Section 3 also examines the issue of mismatch between the model and the actual scanner spectra. Section 4 presents real data results obtained from a table-top cone-beam x-ray system. Section 5 summarizes the results and outlines potential future work.

\section{Polyenergetic x-ray CT}

We model the measurements as independently distributed Poisson ${ }^{2}$ random variables (Erdoğan and Fessler 1999) that are contaminated by extra background counts, caused primarily by scatter. The approach generalizes to more sophisticated models (Fessler et al 2002). Additive detector read-out noise can be accounted for in several ways (Snyder et al 1995). We assume the following statistical model for the measurements:

$$
Y_{i} \sim \text { Poisson }\left\{\int I_{i}(\mathcal{E}) \mathrm{e}^{-\int_{L_{i}} \mu(x, y, \mathcal{E}) \mathrm{d} l} \mathrm{~d} \mathcal{E}+r_{i}\right\} \quad i=1, \ldots, N
$$

where $\mu(x, y, \mathcal{E})$ is the unknown spatially- and energy-dependent attenuation map of the object. The integral in the exponent is taken over the line $L_{i}$ and $I_{i}(\mathcal{E})$ incorporates the energy dependence of both the incident ray source spectrum and the detector sensitivity. The assumed known scatter mean is denoted by $r_{i}$ and $N$ is the number of rays.

\subsection{Object model for polyenergetic $x$-ray $C T$}

We parametrize object space using square pixels. We assume that the object is comprised of $K$ known materials (tissues) and each pixel may contain a mixture of those materials. For any particular tissue, we model the attenuation coefficient as the product of the mass attenuation coefficient and the tissue density (Alvarez and Macovski 1976, Joseph and Spital 1978, Sukovic and Clinthorne 2000). For pixel $j$, the attenuation coefficient is the weighted sum of the attenuation coefficients of all the tissues present in the pixel. Expressed mathematically,

$$
\mu(x, y ; \mathcal{E})=\sum_{j=1}^{p} \mu_{j}(\mathcal{E}) b_{j}(x, y)
$$

2 Recent work showed that x-ray CT measurements are better modelled by the compound Poisson model (Elbakri and Fessler 2003, Whiting 2002). We use the Poisson likelihood here for the sake of simplicity. 
where $b_{j}(x, y)$ is the square-pixel basis function and $\mu_{j}(\mathcal{E})$ is the unknown energy-dependent attenuation coefficient of the materials in pixel $j$. Expressing $\mu_{j}(\mathcal{E})$ using the mass attenuation coefficient/density model gives

$$
\mu_{j}(\mathcal{E})=m(\mathcal{E}) \rho_{j}=\sum_{k=1}^{K} m_{k}(\mathcal{E}) f_{k}^{j} \rho_{j}
$$

where $\rho_{j}$ is the density of pixel $j,\left\{m_{k}(\mathcal{E})\right\}_{k=1}^{K}$ are the mass attenuation coefficients of the $K$ tissues that constitute the object and $f_{k}^{j}$ is a unitless fraction that describes the contribution of material $k$ to attenuation in pixel $j$. We assume that $\left\{m_{k}(\mathcal{E})\right\}_{k=1}^{K}$ are known functions ${ }^{3}$. With the introduction of the tissue fractions, the number of unknowns appears to be $K \times p$. Often in clinical practice, $K=2$ (bone and soft tissue) so the number of unknowns in the polyenergetic problem is twice that of the monoenergetic problem. This seems inevitable in reconstruction from polyenergetic measurements, since at each pixel the reconstruction algorithm must estimate both density and energy information.

Multi-kVp scanning addresses the increase in the number of unknowns by essentially providing a proportional increase in the information available. Dual-kVp imaging has already shown promising results with iterative reconstruction when the object is assumed to consist of bone and soft tissue only (Fessler et al 2002, Sukovic and Clinthorne 2000). With its promise of lower dose without compromising image quality, statistical iterative reconstruction may eventually make dual-kVp imaging clinically feasible.

Another possible strategy is to assume that each pixel is either completely bone or completely soft tissue, and that a segmentation map of the object is available a priori. This approach is used in the JS method and in our earlier work (Elbakri and Fessler 2002). It works well when a good FBP image of an object with distinct anatomical features is available. The FBP image is used to initialize the iterative algorithm and to provide the segmentation map. This approach would not work for objects containing mineral solutions.

In this paper we propose an alternative strategy that avoids segmentation yet can be applied to a single-kVp scan. Using the principle that high-density voxels tend to be composed of bone, we model the fraction of material $k$ in pixel $j$ as a predetermined function of the density of the pixel:

$$
\mu_{j}(\mathcal{E})=\sum_{k=1}^{K} m_{k}(\mathcal{E}) \rho_{j} f_{k}^{j}\left(\rho_{j}\right) .
$$

By predefining the $\left\{f_{k}^{j}\right\}$ functions, there is only one unknown $\left(\rho_{j}\right)$ per voxel, and reconstruction from a single scan is possible. Denoting the system matrix by $\mathbf{A}=\left\{a_{i j}\right\}$ where $a_{i j}=\int_{L_{i}} b_{j}(x, y) \mathrm{d} l$, we can write the line integral of the attenuation coefficient as

$$
\begin{aligned}
\int_{L_{i}} \mu(x, y ; \mathcal{E}) \mathrm{d} l & =\sum_{j=1}^{p} \sum_{k=1}^{K} m_{k}(\mathcal{E}) \rho_{j} f_{k}^{j}\left(\rho_{j}\right) \int_{L_{i}} b_{j}(x, y) \mathrm{d} l \\
& =\sum_{j=1}^{p} \sum_{k=1}^{K} m_{k}(\mathcal{E}) \rho_{j} f_{k}^{j}\left(\rho_{j}\right) a_{i j} .
\end{aligned}
$$

We make the following definitions:

$$
s_{i}^{k}(\rho) \triangleq \sum_{j=1}^{p} a_{i j} f_{k}^{j}\left(\rho_{j}\right) \rho_{j}
$$

3 Data available for anatomical tissues and other materials from Hubbell and Seltzer (1997). 


$$
\underline{s}_{i}(\rho)=\left(s_{i}^{1}, s_{i}^{2}, \ldots, s_{i}^{K}\right)
$$

where $s_{i}^{k}$ denotes the contribution of tissue $k$ to the line integral along the $i$ th ray ${ }^{4}$. From (1), (3) and the definitions above, the mean of the measured data along path $L_{i}$ is

$$
\begin{aligned}
E\left[Y_{i} \mid \rho\right] & =\int I_{i}(\mathcal{E}) \exp \left(-\sum_{k=1}^{K} m_{k}(\mathcal{E}) s_{i}^{k}(\rho)\right) \mathrm{d} \mathcal{E}+r_{i} \\
& =\int I_{i}(\mathcal{E}) \mathrm{e}^{-\underline{m}^{\prime}(\mathcal{E}) \underline{s}_{i}(\rho)} \mathrm{d} \mathcal{E}+r_{i} \triangleq \bar{Y}_{i}\left(\underline{s}_{i}(\rho)\right)+r_{i}
\end{aligned}
$$

where $\underline{m}^{\prime}(\mathcal{E})=\left[m_{1}(\mathcal{E}), \ldots, m_{K}(\mathcal{E})\right]$. We have expressed the mean measurements as a function of the vector $\underline{s}_{i}$ which has as its elements the line integrals of the $K$ different material densities. Because $\bar{Y}_{i}\left(\underline{s}_{i}(\rho)\right)$ is strongly nonlinear, it is easier to work in the log domain. Define

$$
F_{i}\left(\underline{s}_{i}(\rho)\right) \triangleq-\log \frac{\bar{Y}_{i}\left(\underline{s}_{i}(\rho)\right)}{I_{i}}
$$

where $I_{i} \triangleq \int I_{i}(\mathcal{E}) \mathrm{d} \mathcal{E}$. For monoenergetic x-rays, $F_{i}$ would be linear in $\underline{s}_{i}$. For polyenergetic x-rays, the departure of $F_{i}$ from linearity depends on the density and thickness of the object scanned and the range of x-ray energies. Rewrite (8) using $F_{i}$ gives

$$
E\left[Y_{i} \mid \rho\right]=I_{i} \mathrm{e}^{-F_{i}\left(\underline{s}_{i}(\rho)\right)}+r_{i} .
$$

From knowledge of the x-ray spectrum or equivalent measurements, we can determine $F_{i}(\cdot)$ and its gradient, often in the form of a polynomial approximation (Cardinal and Fenster 1990). We next discuss the tissue fraction functions $\left\{f_{k}^{j}\left(\rho_{j}\right)\right\}$.

\subsection{Tissue fraction functions}

The function $f_{k}^{j}\left(\rho_{j}\right)$ specifies a model for the fraction of the $k$ th material in pixel $j$. The actual tissue fraction may depend on more than the pixel density. Ideally, we would want to estimate tissue fractions independently of density or any other parameter. However, that is apparently not possible without additional measurements, as pointed out in the previous section. It is therefore necessary to make model assumptions and impose constraints. The constraint imposed by the JS method and in Elbakri and Fessler (2002) is that each $f_{k}^{j}\left(\rho_{j}\right)$ is either 1 or 0 as specified by the FBP segmentation. Our goal is to improve upon that model.

Although not necessary, we require $f_{k}^{j}\left(\rho_{j}\right) \geqslant 0$ and $\sum_{k} f_{k}^{j}\left(\rho_{j}\right)=1$ for simplicity. The crux of our beam hardening model lies in our choice of the tissue fraction functions. To design reasonable models that are as consistent as possible with attenuation physics, we examine the $\mathrm{x}$-ray spectral characteristics of human tissues.

Figure 1 illustrates the normalized mass attenuation coefficients of several human tissues in the diagnostic x-ray energy range (Hubbell and Seltzer 1997). Several tissues (ovary, brain, muscle, lung, blood) have spectral characteristics that are almost identical to those of water. Fat and breast tissue differ somewhat from water, and bone is significantly different. All soft tissues, including fat and breast, have densities in the range $0.95-1.06 \mathrm{~g} \mathrm{cc}^{-1}$. Because soft tissues are spectrally similar to water, and have densities close to that of water, it is reasonable to use water as a base substance. Cortical bone is another natural choice for a base substance. The model could be augmented to include more base

\footnotetext{
4 In our implementation, we compute the projection along each ray by computing the area of overlap between the ray and pixels in its path normalized by the beam width, so $a_{i j}$ has units of length. The term $s_{i}^{k}$ has units $\mathrm{g} \mathrm{cm}-2$ and is, in some sense, an area density.
} 


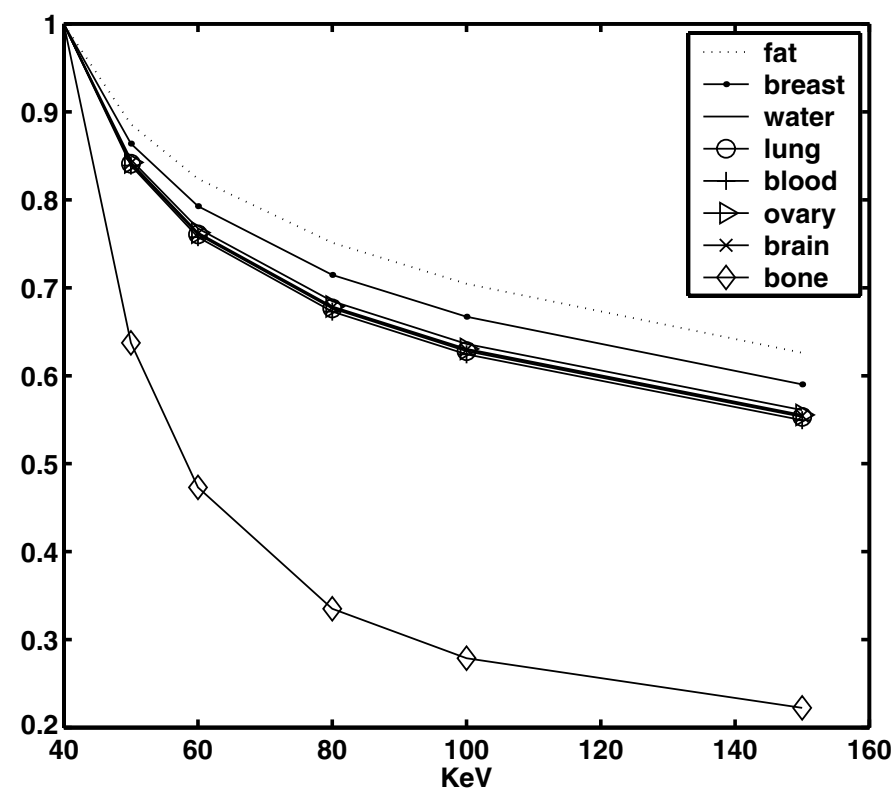

Figure 1. Mass attenuation coefficient of human tissues normalized at $40 \mathrm{keV}$.

substances such as iodine. We use $K=2$ in (3) (water and bone) and model the attenuation coefficient of tissue as follows:

$$
\mu_{j}(\mathcal{E}) \approx\left(m_{w}(\mathcal{E}) f_{w}^{j}\left(\rho_{j}\right)+m_{b}(\mathcal{E}) f_{b}^{j}\left(\rho_{j}\right)\right) \rho_{j}
$$

where $m_{w}(\mathcal{E})$ and $m_{b}(\mathcal{E})$ are the mass attenuation coefficients of the first (water) and second (bone) base substances. The pixel tissue fraction functions $f_{w}^{j}$ and $f_{b}^{j}$ determine to what extent the tissue in pixel $j$ is spectrally water-like or bone-like, depending on the tissue density.

To gain more confidence in our model and insight into possible forms of $f_{w}^{j}$ and $f_{b}^{j}$ we used weighted (by a typical spectrum) least squares to compute the coefficients $f_{w}$ and $f_{b}$ in

$$
m(\mathcal{E})=m_{w}(\mathcal{E}) f_{w}+m_{b}(\mathcal{E}) f_{b}
$$

for the biological substances listed in figure 1. We also imposed the constraints that the solutions be in the interval $[0,1]$, in addition to $f_{w}+f_{b}=1$. The results are listed in table 1 . From table 1, we observe that the water coefficient $f_{w}$ is almost 1.0 for soft tissues and 0.0 for bone, reaffirming that soft tissues are spectrally similar to water and that water and bone can be used as distinct base substances. The pluses and circles in figure 2 illustrate the water and bone coefficients of the different tissues, respectively.

One possible scheme that emerges from examining figure 2 is to set $f_{w}(\rho)=1$ when the density is less than some threshold value and 0 otherwise. This would be closely related to our earlier approach, where pixels are forced to be either bone or water and cannot contain mixtures of tissues (Elbakri and Fessler 2002).

Another approach would be to linearly interpolate the $f_{k}^{j}\left(\rho_{j}\right)$ functions between the water (density $1.0 \mathrm{~g} \mathrm{cc}^{-1}$ ) and bone (density $1.92 \mathrm{~g} \mathrm{cc}^{-1}$ ) coefficients. This implies that the energy dependences of substances between water and bone are linear combinations of the energy dependence of bone and water. This is similar to the approach of De Man et al (2001). The disadvantage of the piece-wise linear approach lies in the fact that many tissue types do not fit this spectral linear combination model. In fact, table 1 shows that soft tissues are better spectrally modelled as equivalent to water. Also, the derivatives of $f_{k}^{j}\left(\rho_{j}\right)$ would have complicating discontinuities in the piecewise linear approach. 


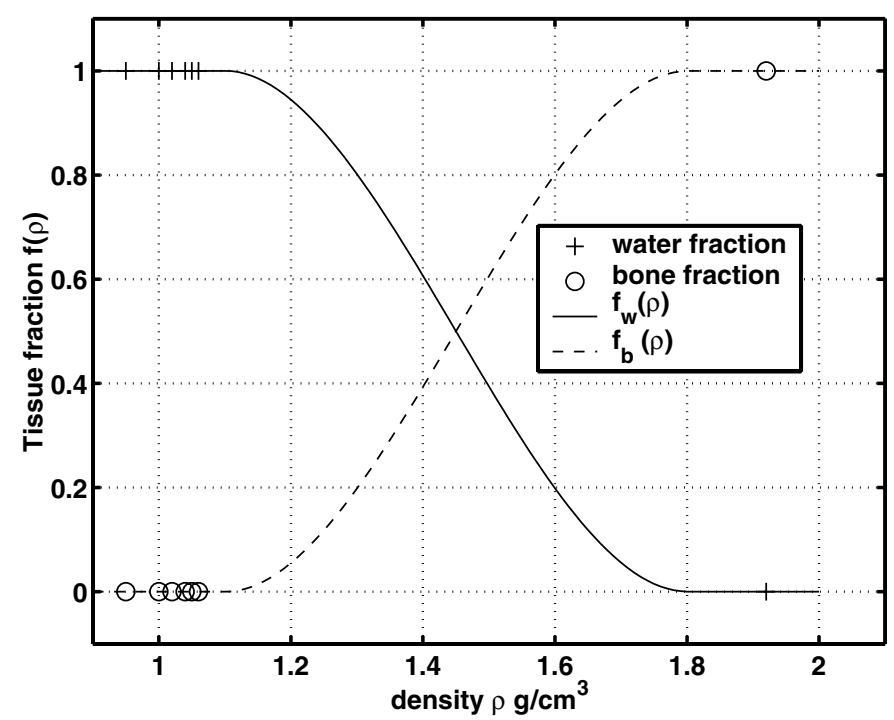

Figure 2. Water and bone fractions computed using constrained least-squares fit to (12), and the displacement model third-order polynomial tissue fraction functions $f_{w}(\rho), f_{b}(\rho)$.

Table 1. Densities and water fractions of human tissues. Most soft tissues have densities close to that of water. The water fraction $f_{w}$ is computed from a weighted least-squares fit to (12).

\begin{tabular}{lll}
\hline Tissue & Density $\left(\mathrm{g} \mathrm{cc}^{-1}\right)$ & $f_{w}$ \\
\hline Fat & 0.95 & 1.0 \\
Breast & 1.02 & 1.0 \\
Water & 1.0 & 1.0 \\
Lung & 1.05 & 0.99 \\
Blood & 1.06 & 0.99 \\
Ovary & 1.05 & 0.99 \\
Brain & 1.04 & 0.99 \\
Muscle & 1.05 & 1.0 \\
Eye lens & 1.07 & 1.0 \\
Testis & 1.04 & 1.0 \\
Cortical bone & 1.92 & 0.0 \\
\hline
\end{tabular}

2.2.1. Displacement model. To model mixed pixels, we propose using the functions plotted in figure 2 using the solid and dotted lines. These are third-order polynomial functions of the tissue density. They have continuous first- and second-order derivatives, and satisfy $f_{w}(\rho)+f_{b}(\rho)=1$. In essence, this choice models tissues that have densities close to that of water and that are spectrally similar to water as 'dense water'. The model allows tissue density to vary from that of water, while assuming it is spectrally equivalent to water. This approach avoids the technical complications posed by the derivatives of piecewise linear functions, and has the potential for better accuracy, particularly for tissues that are spectrally similar to water but have densities larger than water.

The exact shape of the smooth curves is subjective and may be situation dependent. If it were known that an object contained material with density in the mid-range of say, 1.3-1.6 $\mathrm{g} \mathrm{cc}^{-1}$, the curves can be altered so that the algorithm can correctly account for 
that material. The polynomials in figure 2 work well for anatomical structures such as the phantom shown in figure 4.

The polynomials shown in figure 2 do not match the partial volume fractions for tissue mixtures, i.e, they are not mass fractions. For example, a $50 \%$ by mass mixture of bone and water would have a density of $1.315 \mathrm{~g} \mathrm{cc}^{-1}$. Mixtures of other soft tissues with bone would have different $50 \%$ density points and their mass fractions would be different functions of density. To estimate mixtures accurately, an algorithm would have to determine what mixture(s) a particular pixel contains and then parametrize the mass fractions accordingly. This is a prohibitively complex problem. Our major concern in designing the polynomials of figure 2 was to model the spectral properties of homogeneous soft-tissue pixels as equivalent to water. Accurately determining the densities of pixels on tissue boundaries, inherently limited by system resolution, was a secondary consideration. In situations where higher mixture accuracy is desired, the tissue fraction functions can be redesigned to match the mass fractions over a limited density range (Elbakri 2003).

We call this model the displacement model, because it is designed for situations where materials occupy distinct spatial regions, with mixed pixels arising mostly at the boundaries. This model is appropriate for regions of the body with dense and thick skeletal bones. Cortical bone is only about $12 \%$ water (by mass) and it contains significant concentrations high $\mathrm{Z}$ materials $(Z \geqslant 8)(22.5 \% \mathrm{Ca}, 10.3 \% \mathrm{P}$, by mass) (Woodard and White 1986$)$. We therefore treat it as a distinct tissue type. This is possible because its density value is also far enough from that of water. Other kinds of bones, on the other hand, are better described as mineral solutions in water, with mineral composition of a few per cents (Woodard and White 1986).

2.2.2. Solution model. We also consider another approach that we call the solution model, where we assume the object contains a mineral solution in water (or some other solvent). In this model, the water-mineral solution density is greater than or equal to the density of water, and the attenuation coefficient can be modelled as follows:

$\mu(\mathcal{E}) \approx m_{w}(\mathcal{E}) \rho_{w}+m_{b}(\mathcal{E})\left(\rho-\rho_{w}\right)=\rho\left(m_{w}(\mathcal{E}) \frac{\rho_{w}}{\rho}+m_{b}(\mathcal{E})\left(1-\frac{\rho_{w}}{\rho}\right)\right)$

for $\rho>\rho_{w}$. The fraction functions for this model are shown in figure 3 and are given by

$$
\begin{aligned}
& f_{w}(\rho)=\left\{\begin{array}{lll}
1 & \text { if } & \rho \leqslant \rho_{w} \\
\frac{\rho_{w}}{\rho} & \text { if } & \rho>\rho_{w}
\end{array}\right. \\
& f_{b}(\rho)=\left\{\begin{array}{lll}
0 & \text { if } & \rho \leqslant \rho_{w} \\
1-\frac{\rho_{w}}{\rho} & \text { if } & \rho>\rho_{w}
\end{array}=1-f_{w}(\rho)\right.
\end{aligned}
$$

where $\rho_{w}$ is the density of water (or, more generally, the solvent). This model effectively assumes that the object consists of water everywhere, whose density (in some regions) has increased because of mineral content. The fraction functions in this model are the mass fractions of water and mineral in the solution. This model is designed for cases when bone is not very dense, and is more like a water-calcium solution than solid bone mineral. One advantage of this approach is that it makes no assumptions regarding the density of the mineral.

The solution model ignores what is known to chemists as the displacement effect ${ }^{5}$ which arises because the amount of water per cc varies nonlinearly with mineral concentration (Cody and Flynn 1989, Rao et al 1987). In other words, the physical density of a $100 \mathrm{mg} \mathrm{ml}^{-1}$ solution will not be exactly equal to $1.1 \mathrm{~g} \mathrm{cc}^{-1}$. This effect is small at low concentrations. Although it is possible to reparameterize (13) to account for this effect, we do

5 Not to be confused with the displacement model discussed in the previous section. 


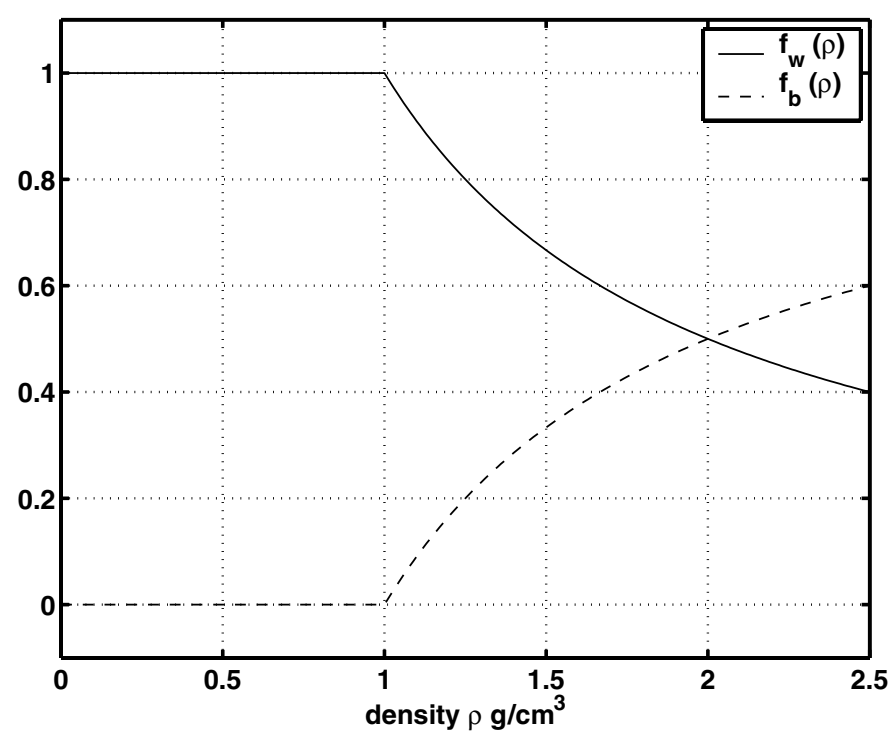

Figure 3. Solution model tissue fraction functions $f_{w}(\rho), f_{b}(\rho)$. The fractions in this model represent the mass fractions of water and mineral as explained in (15).

not incorporate it at this point. We can correct for the error introduced by ignoring the effect of displacement in solution by applying a simple polynomial correction, based on empirical measurements of density versus concentration, to an image reconstructed with the proposed solution model (Elbakri 2003).

We developed this method in hindsight as we tried to apply our algorithm to reconstruct real data generated from a phantom consisting of potassium phosphate solutions of different concentrations. This approach gave better quantitative results than the displacement model (see section 4).

\subsection{Problem statement}

After choosing one of the models for the fractions $f_{k}^{j}(\rho)$, the reconstruction problem becomes estimating the density vector $\rho$ from the measurements, using the model (10). We use a likelihood-based estimation approach. The negative log-likelihood for independent Poisson measurements is given by

$$
-L(\rho)=\sum_{i=1}^{N} E\left[Y_{i} \mid \rho\right]-Y_{i} \log \left(E\left[Y_{i} \mid \rho\right]\right)
$$

where $E\left[Y_{i} \mid \rho\right]$ is given by (8). When the system matrix $\mathbf{A}$ has full column rank and the data is noise-free, minimizing the negative likelihood would give a perfect result. In reality, the data are noisy and maximum likelihood (ML) will give a very noisy reconstruction due to the ill-posedness of the problem, hence the need for regularization.

We regularize by adding a penalty term to the likelihood function. We consider pairwise regularization of the following form:

$$
R(\mu)=\sum_{j=1}^{p} \sum_{k \in \mathcal{N}_{j}} \psi\left(\mu_{j}-\mu_{k}\right)
$$


where $\psi$ is a potential function and $\mathcal{N}_{j}$ is some neighbourhood of pixel $j$. For our simulation and experimental results, we used the convex edge-preserving Huber penalty

$$
\psi(x ; \delta)= \begin{cases}\frac{x^{2}}{2} & x<\delta \\ \delta|x|-\frac{\delta^{2}}{2} & x \geqslant \delta .\end{cases}
$$

Combining the likelihood with a penalty gives a penalized-likelihood (PL) cost function

$$
\Phi(\rho)=-L(\rho)+\beta R(\rho)
$$

where $\beta$ is a scalar that controls the tradeoff between the data-fit and the penalty terms. The goal of the reconstruction technique becomes to minimize (19) subject to certain object constraints such as non-negativity:

$$
\hat{\rho}=\underset{\rho \geqslant 0}{\operatorname{argmin}} \Phi(\rho) .
$$

The next section describes an iterative technique for solving (20) approximately.

\subsection{Polyenergetic $x$-ray $C T$ iterative algorithm}

The goal of the iterative algorithm is to find the minimizer in (20). The optimization transfer principle (De Pierro 1993, 1995, Elbakri and Fessler 2002, Erdoğan and Fessler 1999) has proved to be a very effective tool in simplifying such problems. Stated simply, optimization transfer enables us to replace a complex likelihood like (19) with surrogate cost functions that are simpler and easier to minimize. Optimization transfer methods can ensure that the cost function decreases monotonically each iteration. Because the Poisson likelihood includes a scatter term $\left(r_{i}\right)$ and because of the nonlinearity of $F_{i}(s)$, the likelihood is not convex, so monotonicity is all that can be claimed about the convergence of the algorithm.

Along the lines of our earlier work (Elbakri and Fessler 2002), we apply successive surrogates to the penalized likelihood cost function, ultimately resulting in a cost function that is quadratic and separable in $\rho$. Quadratic functions are more easily minimized, and separability allows the algorithm to update all pixels simultaneously.

We omit the details in this paper and refer the reader to Elbakri and Fessler (2002). The resulting algorithm is a diagonally-preconditioned gradient descent method of the following form:

$$
\rho^{n+1}=\left[\rho^{n}-\mathbf{D}^{-1} \nabla \Phi\left(\rho^{n}\right)\right]_{+}
$$

where $\nabla \Phi\left(\rho^{n}\right)$ is the gradient of the cost function, $\mathbf{D}$ is a diagonal matrix that influences the rate of convergence and and monotonicity of the algorithm, and $[\cdot]_{+}$enforces the nonnegativity constraint. For a monotonic algorithm, the elements of $\mathbf{D}$ must be such that a surrogate satisfies the conditions of optimization transfer. It is possible to derive a version of $\mathbf{D}$ that gives a monotone algorithm, but the elements of the matrix will have to be updated at every iteration, resulting in a more computationally expensive approach (Elbakri 2003). Since monotonicity will be compromised anyway by using ordered subsets to accelerate the algorithm, a precomputed approximate $\mathbf{D}$ will suffice. By making certain assumptions about the object (Elbakri and Fessler 2002), we derive an approximate precomputed 'curvature' matrix using the second derivative of the quadratic surrogate evaluated at the initial image:

$$
d_{j}=m_{w}^{2}\left(\mathcal{E}_{\text {eff }}\right) \sum_{i=1}^{N} a_{i j}^{2} Y_{i}
$$




\section{True Object}

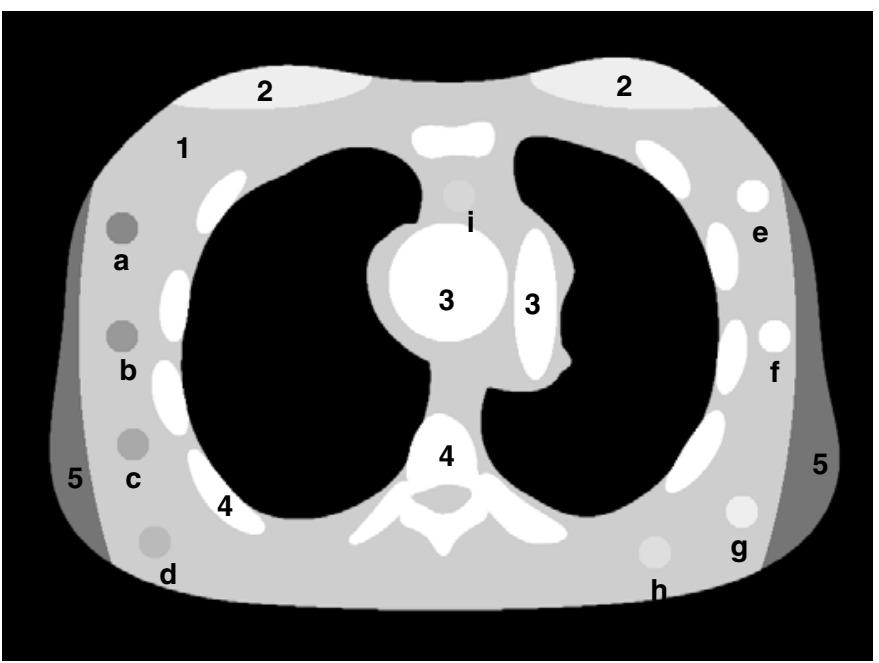

Figure 4. Simulation experiment. True $1024 \times 1024$ object containing soft tissue (1), breast (2), blood (3), bone (4) and fat (5). The water lesions are (a) $-4 \%$, (b) $-3 \%$, (c) $-2 \%$, (d) $-1 \%$, (e) $-6 \%$, (f) $3 \%$, (g) $2 \%$, (h) $1 \%$, (i) $0.5 \%$ different from $1 \mathrm{~g} \mathrm{cc}^{-1}$. Window level $=-45 \mathrm{HU}$ and window width $=150 \mathrm{HU}$.

where $d_{j}$ is the $j$ th entry of the diagonal matrix $\mathbf{D}$ and $\mathcal{E}_{\text {eff }}$ is the effective energy of the x-ray spectrum defined as

$$
\mathcal{E}_{\text {eff }} \triangleq \frac{\int \mathcal{E} I_{i}(\mathcal{E}) \mathrm{d} \mathcal{E}}{\int I_{i}(\mathcal{E}) \mathrm{d} \mathcal{E}} .
$$

With this approximation, the method is not guaranteed to be monotonic. One could check the cost function at each iteration and apply the monotonic update on those rare cases where the cost function does not decrease using the approximate method. In our experience, with a good starting image, such as a FBP reconstruction, the cost function always decreases.

As far as the computational load of the algorithm, each iteration involves one forward projection and one backprojection. An iteration is therefore roughly equivalent to two runs of FBP.

\section{Simulation results}

We assessed the effectiveness of our algorithm with simulated transmission polyenergetic Poisson x-ray projections. We simulated a parallel beam geometry, but the algorithm applies equally well to other geometries. We used a $100 \mathrm{kVp}$ spectrum (incident mean $45 \mathrm{keV}$ and standard deviation $14.8 \mathrm{keV}$ ) using the code available from Boone and Seibert (1997) with $10 \mathrm{~mm}$ aluminium filtration at the source to give a spectrum shape similar to clinical practice.

Figure 4 shows the phantom used. The phantom is based on the NCAT phantom (Tsui et al 1993), where in addition to (water-like) soft tissue and bone, we have manually introduced regions of breast, fat, blood and soft-tissue lesions with contrast differences of $-4 \%$ to $6 \%$. The bones and soft tissue have densities $1.8-2.0 \mathrm{~g} \mathrm{cc}^{-1}$ and $0.2-1.0 \mathrm{~g} \mathrm{cc}^{-1}$ respectively. The 

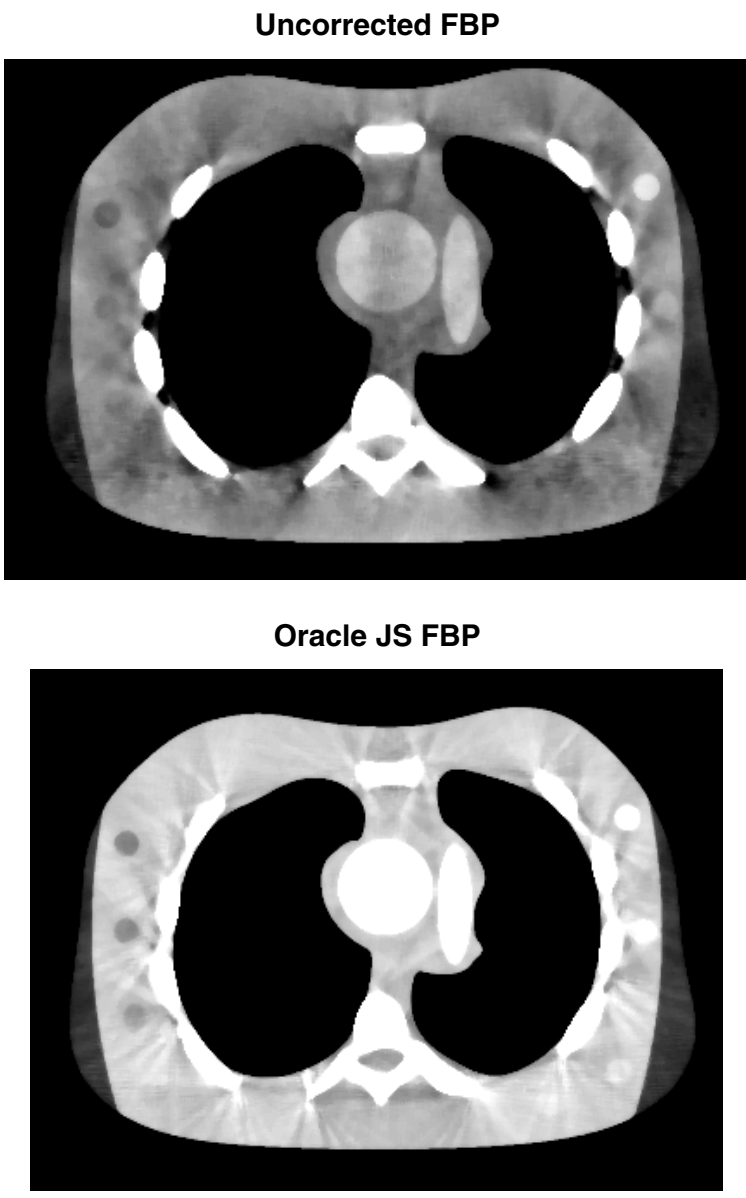

Figure 5. Simulation experiment. Top: uncorrected FBP (scaled by ratio of water density to attenuation coefficient). Bottom: JS correction post processed with a $5 \times 5$ median filter. Window level $=-45 \mathrm{HU}$ and window width $=150 \mathrm{HU}$.

densities for fat, bone and blood are identical to those cited in table 1 . The measurements are simulated using the actual attenuation properties of the different tissues, not just those of water and bone. The sinogram has 1200 radial bins with $0.055 \mathrm{~mm}$ ray spacing and 1200 angular steps over $180^{\circ}$. We did not simulate scatter. The blank scan value was $4.87 \times 10^{6}$ counts/detector. The ray with the lowest SNR had 77 counts. We generated pseudo-random Poisson variates for each sinogram element. The original phantom was $1024 \times$ $1024(0.055 \mathrm{~mm} /$ pixel $)$ but we reconstructed the data on a $512 \times 512$ grid $(0.11 \mathrm{~mm} / \mathrm{pixel})$ to generate the effect of mixed pixels.

The data was reconstructed by uncorrected FBP, JS-corrected FBP, a polyenergetic statistical algorithm with a pre-segmented bone/soft tissue map (Elbakri and Fessler 2002), and the proposed iterative algorithm. We used a Hanning window in all FBP reconstructions, and post-processed the FBP imaged with a $5 \times 5$ median filter. For the JS correction and one implementation of pre-segmented iterative algorithm, we used an idealized oracle segmentation based on the true object. For the oracle segmentation, each $2 \times 2$ block of pixels in the true object was classified as a bone or water pixel for reconstruction using a majority rule. 


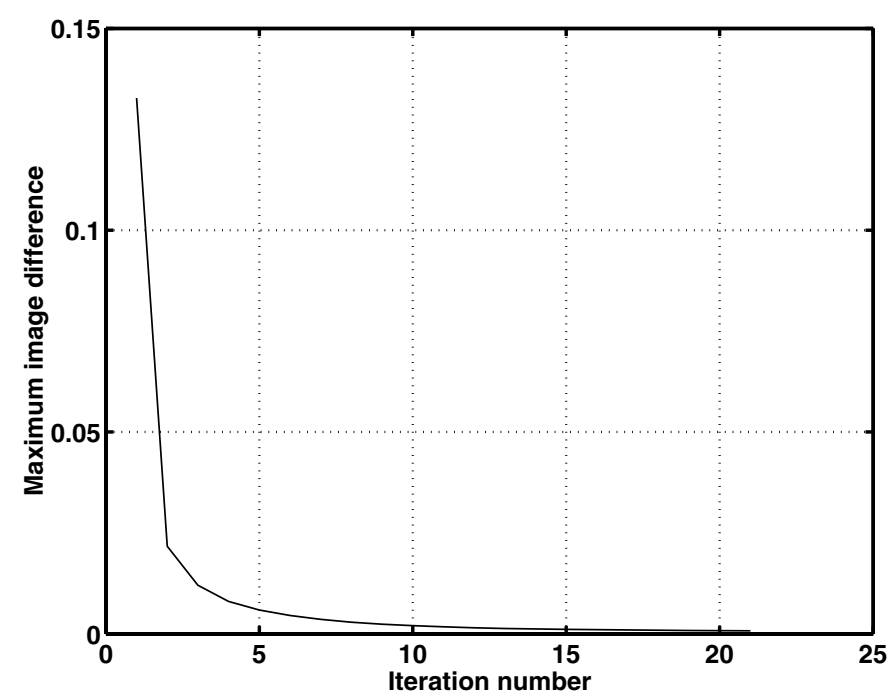

Figure 6. Absolute maximum difference between successive iterations (normalized by the maximum pixel of the current iteration) of the proposed algorithm with simulated data of the phantom in figure 4.

The iterative algorithm in Elbakri and Fessler (2002) accommodates only water and bone, and requires prior knowledge of their distribution in the image. With oracle segmentation, we assume this algorithm has 'perfect' prior knowledge. This is not a practical case, but is included for the sake of comparison to provide an upper bound on performance.

For the iterative methods, we included edge-preserving shift-variant regularization (Fessler and Rogers 1996). The iterative algorithms ran until the absolute normalized maximum difference between successive iterations fell below a prespecified small value. To achieve a maximum normalized difference of less than $2 \times 10^{-3}$, we used 21 iterations with 50 subsets.

\subsection{Image reconstruction results}

Since algorithms with beam hardening correction usually reconstruct density values, we display the reconstructed images using a density Hounsfield unit (HU) defined as

$$
\mathrm{HU}=\frac{\rho-\rho_{\text {water }}}{\rho_{\text {water }}} \times 1000
$$

If one wishes to display attenuation Hounsfield units, the density images can be segmented and each material scaled by its mass attenuation coefficient at some energy.

Figure 5 shows the FBP image reconstruction results. Figure 6 illustrates how the normalized maximum difference decreases with iteration for the proposed iterative algorithm. Figure 7 shows the iterative reconstruction results. The uncorrected FBP image suffered from severe beam hardening artefacts. The statistical iterative reconstructions exhibit significantly better artifact reduction than the Joseph and Spital image because they inherently model the nonlinearity and reconstruct the data accordingly. The segmentation-free algorithm reconstruction is comparable to the oracle pre-segmented iterative reconstruction. None of the reconstruction algorithms produced an image where breast tissue could be visually discerned from the surrounding water. 
Pre-segmented Iterative Image

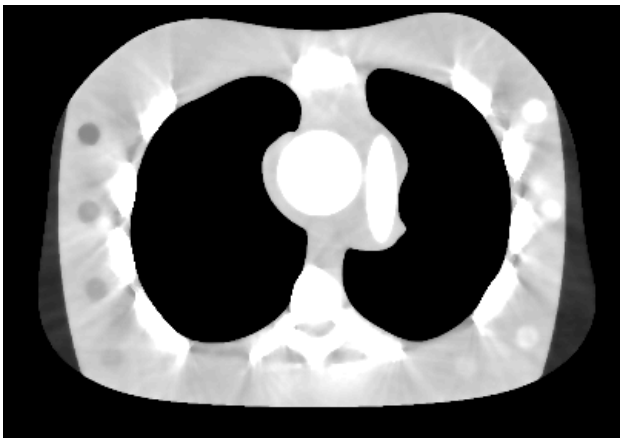

Oracle-segmented Iterative Image

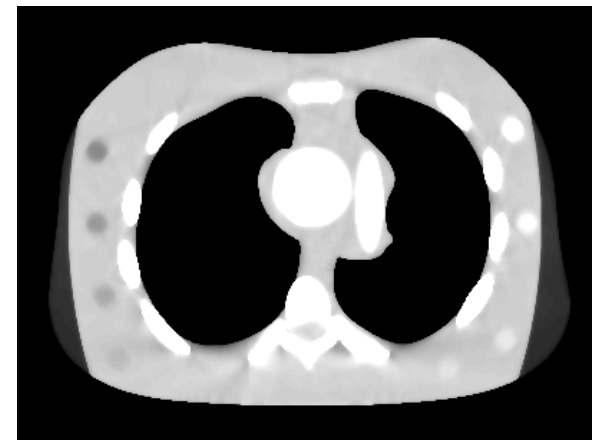

Segmentation-free Iterative Image

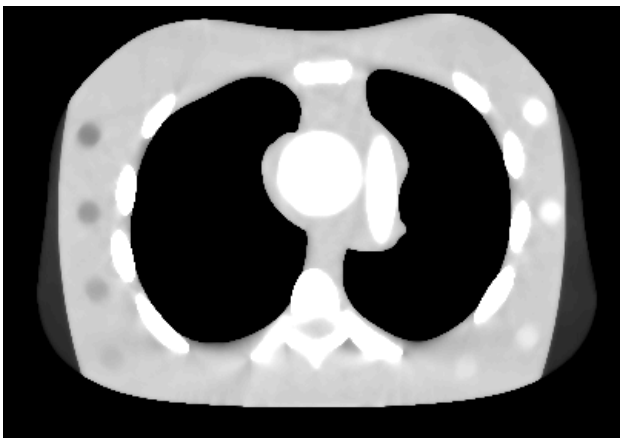

Figure 7. Simulation experiment reconstruction results. Top: pre-segmented iterative algorithm. Middle: Oracle pre-segmented iterative reconstruction. Bottom: proposed statistical reconstruction algorithm. Window level $=-45 \mathrm{HU}$ and window width $=150 \mathrm{HU}$.

In addition to qualitative assessment of artifact reduction, we quantify the performance of the different algorithms using the percent normalized root mean squared error (NRMSE) in uniform regions within each tissue type. The uniform regions were selected far from edges, and contained between 100 and 300 pixels. It is difficult to quantify the performance of the algorithms at tissue boundaries because of the intentional mismatch between image-domain grids used for data generation and reconstruction. 
Table 2. Per cent normalized root mean squared error of the different reconstructions of the altered NCAT phantom in figure 4.

\begin{tabular}{llllll}
\hline Reconstruction method & Water & Fat & Blood & Breast & Bone \\
\hline Oracle JS FBP & 0.6 & 3.8 & 1.1 & 1.6 & 7.4 \\
Pre-segmented iterative & 0.5 & 4.1 & 0.3 & 2.3 & 0.4 \\
Segmentation-free iterative & 0.16 & 4.1 & 0.4 & 2.1 & 0.2 \\
Oracle-segmented iterative & 0.15 & 4.2 & 0.4 & 2.1 & 0.2 \\
\hline
\end{tabular}

Table 2 shows the NRMSE results. The proposed iterative method quantitatively outperforms JS-corrected FBP with oracle segmentation for all tissues except fat and breast. It also has better overall NRMSE performance than the pre-segmented iterative reconstruction, and is comparable in performance to the idealized oracle pre-segmented iterative algorithm image.

Table 2 suggests that the proposed algorithm gives more accurate results than JS FBP and the pre-segmented iterative algorithm for all tissues, except fat and breast tissue. We observed that fat and breast are generally underestimated by the algorithms. Fat is less dense and less attenuating than water. Since the proposed algorithm uses the spectral attenuation properties of water, it is reasonable for the estimated fat density to be less than the true value, to compensate for the higher attenuation introduced by the water model. A similar argument follows for breast tissue. In the case of breast tissue, however, tissue density is very close to that of water, and breast tissue is only slightly less attenuating than water. This explains the difficulty in producing images where breast is visually distinguishable from water. At the effective energy of the $100 \mathrm{kVp}$ spectrum used, the difference in attenuation coefficient between water and breast is less than $1 \%$, corresponding to an attenuation Hounsfield unit difference of $6 \mathrm{HU}$.

Figure 8 illustrates the performance of the different algorithms in terms of low contrast detectibility. The phantom has nine soft tissue lesions with density HU values of $-40,-30,-20,-10,5,10,20,30,60$. The $5 \mathrm{HU}$ lesion, corresponding to a $0.5 \%$ difference from the actual density of water, could not be discerned from any of the reconstructions. The other lesions were visible in both the JS FBP and statistical reconstructions using a narrow display window. Figure 8 shows that the iterative reconstruction images are generally closer to the true contrast levels than the JS FBP image.

\subsection{Robustness to spectrum model mismatch}

Measuring the $\mathrm{x}$-ray source spectrum is a challenging problem that requires careful measurements and calibrations. Measuring the spectrum directly is difficult, and indirect methods are often used. One possibility is to attempt to estimate the function $F_{i}$ in (7) by measuring phantoms of different thicknesses and using polynomial fits on the data. Another approach is to solve for the spectrum from a discretized version of the transmission equation using measurements taken on phantoms with varying thicknesses (Ruth and Joseph 1997 , Yan et al 2000). Regardless, attempts to measure the scanner spectrum are likely to give approximate results. Moreover, the $\mathrm{kVp}$ setting on $\mathrm{x}$-ray scanners may be imperfect, and the spectrum may change with tube drift over time.

It is therefore important to investigate the robustness of our reconstruction method to mismatches between the spectrum that generates data and the assumed spectrum used to tabulate $F_{i}$ and its gradient. 


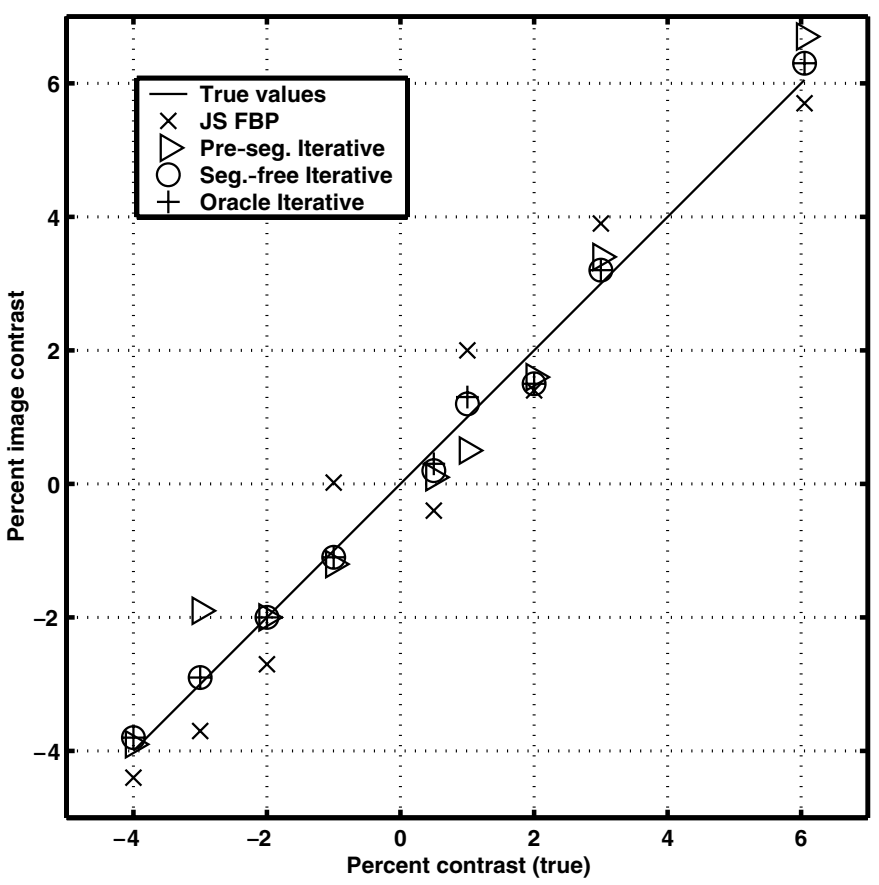

Figure 8. Percent contrast of the soft tissue lesions in the phantom of figure 4 for the different reconstruction algorithms.

We reconstructed $100 \mathrm{kVp}$ data generated from a phantom similar to that described in the previous section, but without the soft-tissue lesions. In addition to the matched $100 \mathrm{kVp}$ algorithm, we used algorithms based on 80, 90, 110 and $120 \mathrm{kVp}$ spectra. The (incident) mean energies of these spectra deviate by $11.2 \%, 5.2 \%, 4.7 \%$ and $9.0 \%$ respectively, from that of the $100 \mathrm{kVp}$ spectrum. This experiment reflects a practical situation where the $\mathrm{kVp}$ setting is inaccurate, because of tube drift or other effects. Figure 9 shows the spectra used.

The images shown in figure 10 were reconstructed using the algorithm parameters listed in the previous section. The only difference between the different reconstructions was the spectrum used to tabulate $F_{i}$. The display window is the same for all the images. Spectral mismatch resulted in images exhibiting different 'brightness' levels, and inaccurate pixel density values, as further illustrated by figure 11. Algorithms based on lower kVp models underestimated density values and vice versa. The error introduced in estimating tissue densities by spectrum deviations is illustrated in figure 12 .

The error introduced by spectral mismatch is generally higher for higher density bones. This is reasonable since dense bones are highly attenuating and the deviation will be increasingly nonlinear. This can also be seen from the scatter plots of figure 13 . The solid lines in figure 13 are the identity lines for density values of the image reconstructed using the correct spectrum. The dotted scatter plots illustrate the deviation of the images reconstructed with mismatched spectra from the matched spectrum case. As the figure illustrates, the deviation is nonlinear and increases with object density, and results in overestimation and underestimation of density values for higher and lower $\mathrm{kVp}$ settings, respectively.

Figure 12 also shows an interesting pattern for fat and breast tissues. Their error patterns are very similar and they have minimum error at $110 \mathrm{kVp}$. Fat and breast tissues have similar attenuation properties (see figure 1), and are less attenuating than water. Since the algorithm 


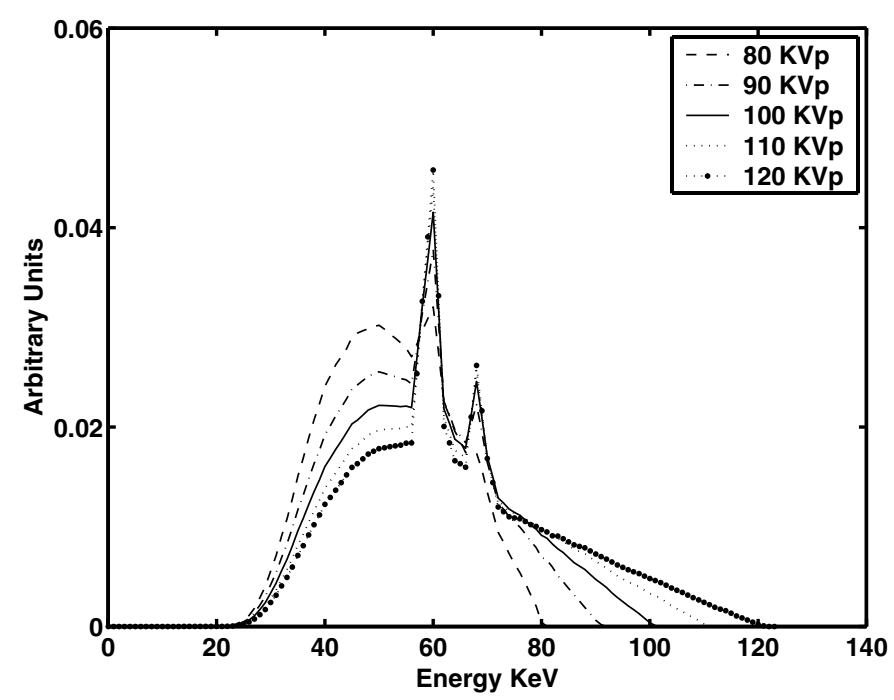

Figure 9. Energy spectra used to examine effect of spectrum mismatch.

(a) $80 \mathrm{kVp}$

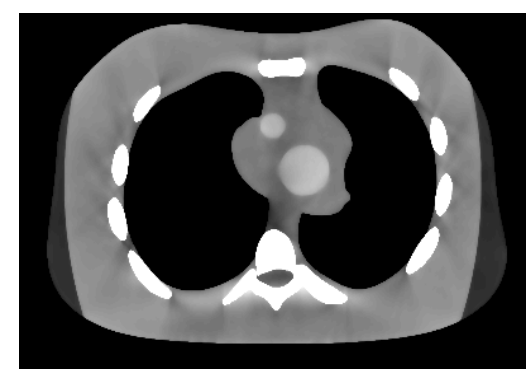

(c) $110 \mathrm{kVp}$

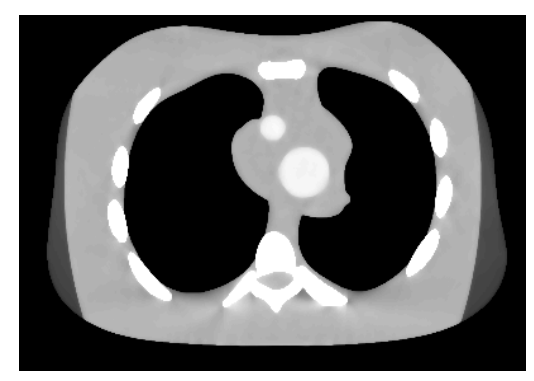

(b) $90 \mathrm{kVp}$

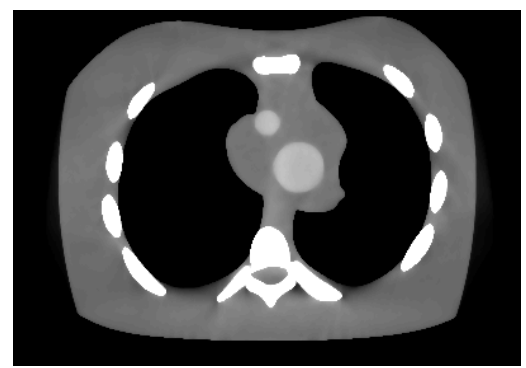

(d) $120 \mathrm{kVp}$

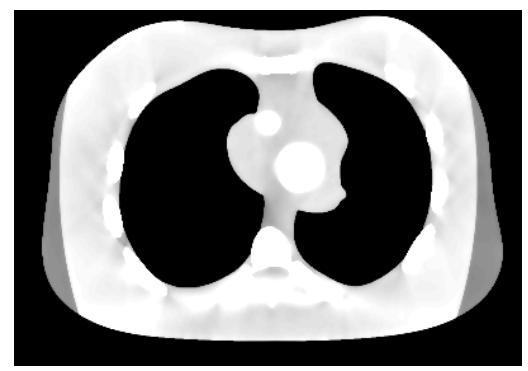

Figure 10. Spectrum mismatch results. $100 \mathrm{kVp}$ data reconstructed with (a) $80 \mathrm{kVp}$ algorithm, (b) $90 \mathrm{kVp}$ algorithm, (c) $110 \mathrm{kVp}$ algorithm, (d) $120 \mathrm{kVp}$ algorithm. WL $=-25 \mathrm{HU}, \mathrm{WW}=$ $250 \mathrm{HU}$.

with exact spectrum underestimates fat, it is reasonable that a higher $\mathrm{kVp}$ setting that tends to overestimate tissue densities will give less error. Note also that blood and water, which have very similar attenuation properties, have similar error patterns. 


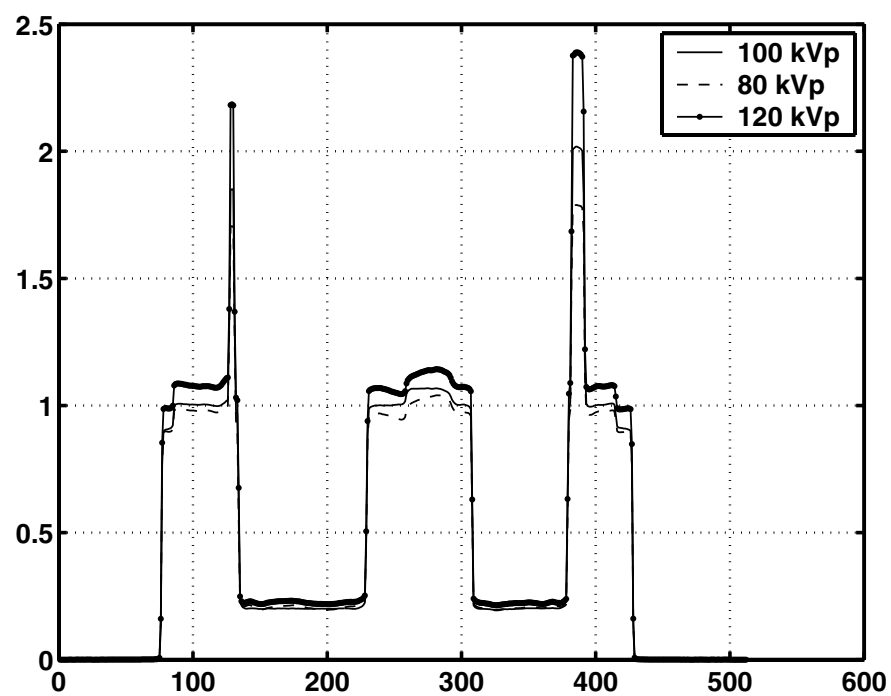

Figure 11. Spectrum mismatch results. Profile plots of one row of the reconstructed images. The plots show that a lower $\mathrm{kVp}$ mismatch causes the algorithm to underestimate tissue density. Higher $\mathrm{kVp}$ mismatch has the opposite effect.

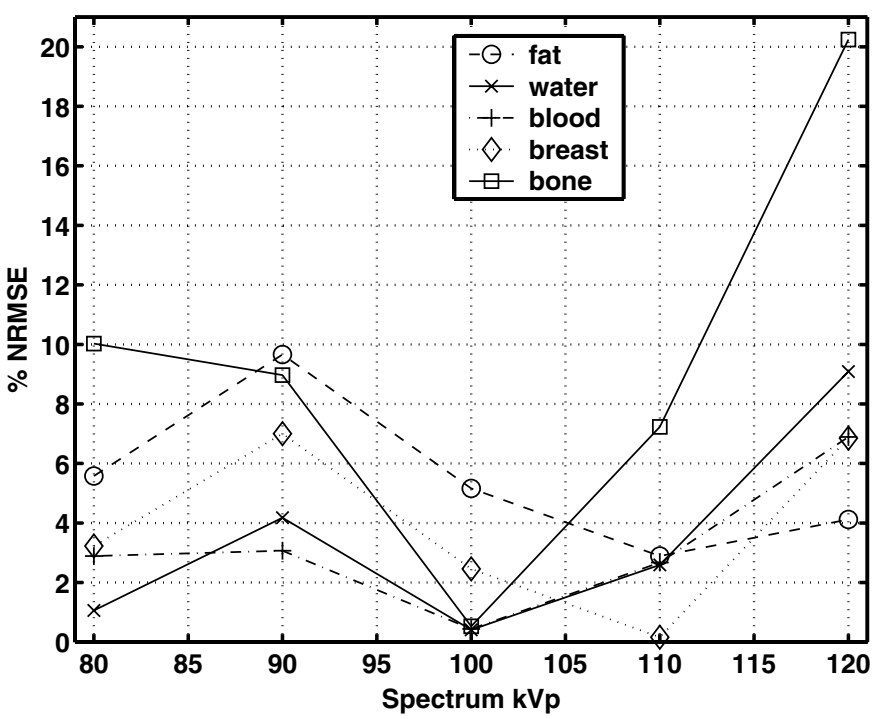

Figure 12. Normalized root mean squared error for different tissues of the phantom caused by different $\mathrm{kVp}$ settings.

The acceptable degree of deviation between the actual and assumed spectra will depend on the application. We note that the density errors are closely related to the percent errors of the tube potentials. Clearly, in quantitative applications, as close a match as possible is desirable. If the application requires only visual and qualitative assessments, then a mismatch of $20 \mathrm{kV}$ in the $\mathrm{kVp}$ scanner setting may be tolerable. 

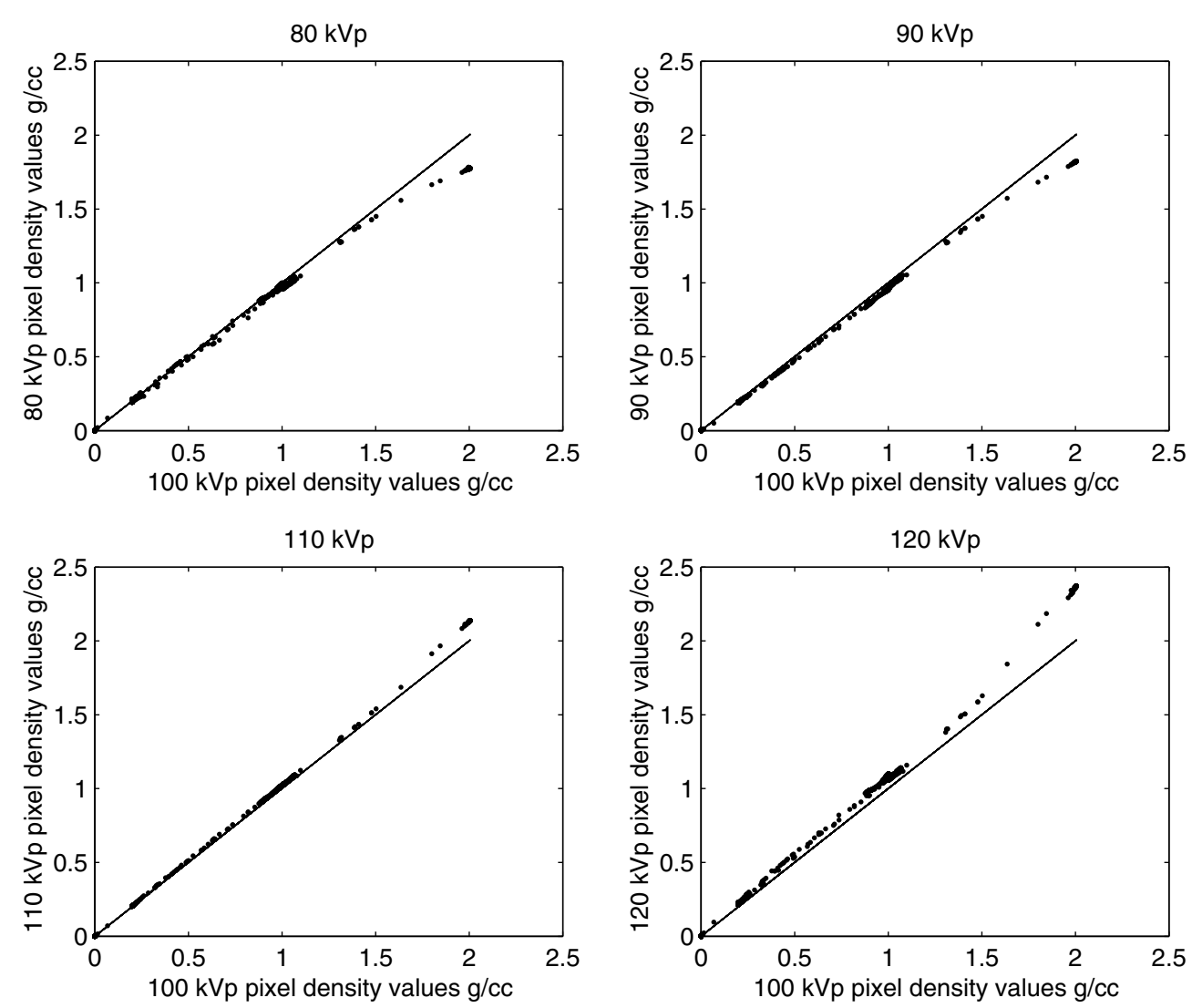

Figure 13. Spectrum mismatch scatter plot. The solid line (-) is the identity line of the density values of the image reconstructed with a perfectly matched spectrum. The dotted lines are scatter plots that illustrate the deviations caused by spectrum mismatch.

\section{Real data results}

To validate our method with real data, we obtained real transmission data acquired on the table-top system described in Sukovic and Clinthorne (1999). The phantom consisted of a water cylinder (15 $\mathrm{cm}$ in diameter) with five embedded smaller cylinders $(2.5 \mathrm{~cm}$ diameter) filled with different concentrations of (dry) potassium phosphate dibasic $\left(\mathrm{K}_{2} \mathrm{HPO}_{4}\right)$ solution. The powder was mixed with water to form the following solutions: 50, 100, 141, 150 an $200 \mathrm{mg} \mathrm{ml}^{-1}$, corresponding to physical densities ${ }^{6}$ of $1.04,1.08,1.108,1.115$ and $1.153 \mathrm{~g} \mathrm{cc}^{-1}$ respectively. This powder is quite soluble in water, so we used the solution model (14) and (15) for image reconstruction. This phantom was intended to mimic a range of trabecular bone densities.

Fan-beam data (834 angular view, 576 detector bins) were collected at $60 \mathrm{kVp}$ using a $360^{\circ}$ scan with axial collimation and no scatter grid. The beam was filtered with $1 \mathrm{~mm}$ aluminium and $0.5 \mathrm{~mm}$ copper and the spectrum estimated from Monte Carlo simulations. The measured transmission sinogram is shown in figure 14 with a narrow display window to illustrate fine details. The data was reconstructed on $256 \times 256$ grids using the proposed

6 These densities were not measured directly, but were calculated based on data in Cody and Flynn (1989) and Rao et al (1987). 


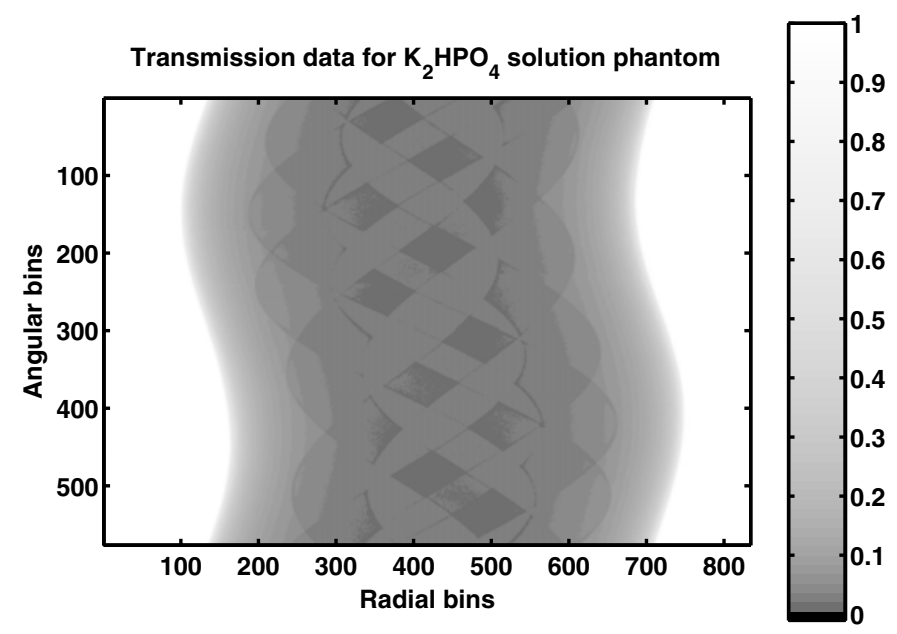

Figure 14. Measured sinograms. Note that the display range has been warped to better illustrate details.

polyenergetic statistical algorithms (10 iterations, 36 subsets) with both the displacement and solution models, as well as water-corrected FBP. We applied a polynomial correction to the solution model image to account for the displacement effect in solution. The polynomial coefficients were computed to fit density and concentration data reported for $\mathrm{K}_{2} \mathrm{HPO}_{4}$ in Cody and Flynn (1989) and Rao et al (1987).

\subsection{Scatter estimation}

Initial reconstruction suffered from streaking that was typical of the kind caused by scatter, but no scatter estimate was available with the data. To estimate scatter, we computed a difference sinogram between the real sinogram and a synthetic sinogram corresponding to a numerical water phantom based on a segmentation of an initial reconstruction. We excluded sinogram rays passing through the potassium phosphate regions from the difference sinogram and replaced them with interpolated values. We applied smoothing to the difference sinogram to estimate scatter. Figure 15 shows the estimated scatter sinogram. We estimate scatter at about $1 \%$ of the overall measured signal.

The shape and value of the estimated scatter sinogram are similar to those in earlier reports (Glover 1982, Joseph and Spital 1982). This is not a fully developed scatter estimation/correction technique. Rather, it is a heuristic approach to help verify that scatter is the likely source of the observed artifact and to correct for it in the absence of any information on the scatter properties of the system.

\subsection{Results and comparisons}

Figure 16 shows the polyenergetic iterative reconstructed image. We do not show the FBP reconstruction because this phantom has no high-density regions that cause severe beam hardening streaking, and the images are visually comparable. We do, however, compare the quantitative accuracy of our approach with FBP in determining the density of the different $\mathrm{K}_{2} \mathrm{HPO}_{4}$ concentrations in figure 17 . We also compare the accuracy of the displacement and solution object models in figure 17. The apparent roughness of the edges of the phantom 


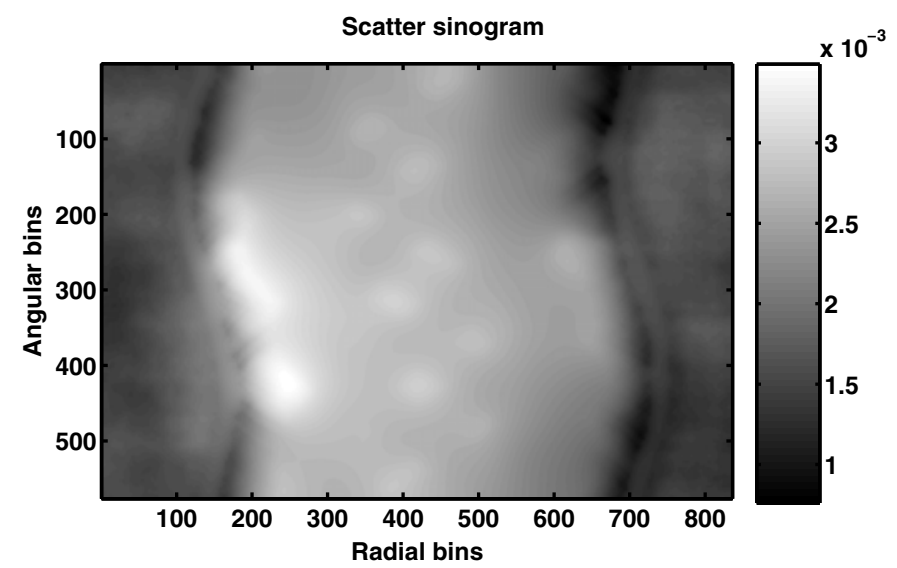

Figure 15. Estimated scatter sinogram obtained by smoothing the difference between an idealized sinogram and the real sinogram.
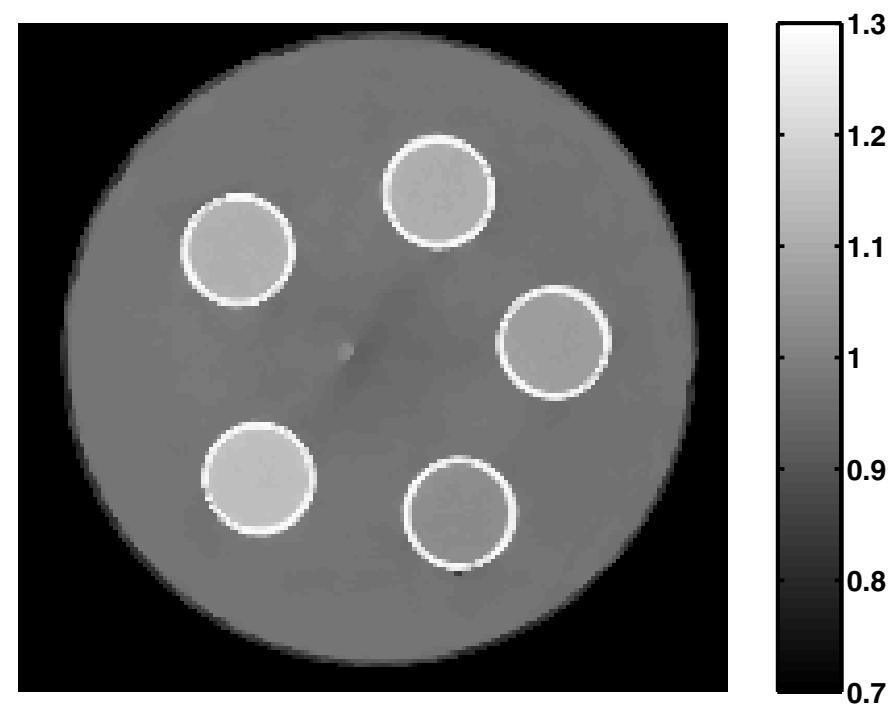

Figure 16. Iterative reconstruction of real data using 10 iterations and 36 subsets with an edgepreserving penalty.

image in figure 16 is due to the algorithm attempting to reconstruct the walls of the plastic container in the phantom.

Although both the displacement and solution models give good visual results, the solution model has significantly higher quantitative accuracy. The solution model polyenergetic statistical reconstruction has the best accuracy of all methods, with maximum error of $-1.2 \%$ ( $2.7 \%$ error before post-correction for water displacement). The values in figure 17 are the averages of uniform regions inside each cylinder. Trying to address the apparent bias present in the potassium phosphate cylinders in the displacement model reconstruction was the motivation behind deriving the solution model. 


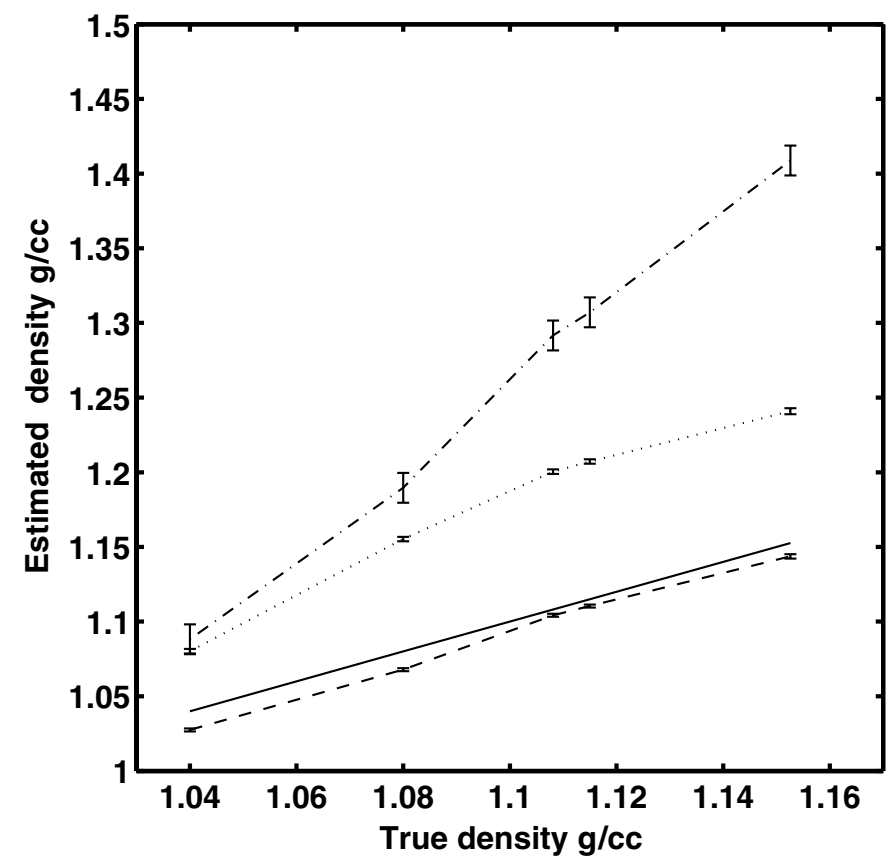

Figure 17. Accuracy of the different reconstruction techniques. Figure key: true values (-); solution model (-- ) ; displacement model (..); water-corrected FBP (-. - .) . The solution model reconstruction values are very close to the true values (max. error $-1.2 \%)$. The displacement model has errors on the order of $8 \%$. The solution model image was post-corrected for the displacement effect. The error bars represent the width of one standard deviation of each measurement.

\section{Conclusions}

We have introduced a statistical iterative reconstruction algorithm for energy dependent $\mathrm{x}$ ray attenuation that produces images with significantly reduced beam hardening artefacts, and we have demonstrated its effectiveness for objects of bone and soft tissue and mineral solutions. Unlike most other transmission CT iterative algorithms, our algorithm is based on a realistic polyenergetic model and has been extended to allow for mixed pixels and mineral solutions. The algorithm we present requires knowledge of the incident spectrum or tabulated measurements of the line integrals of two base substances. The statistical algorithm minimizes a separable paraboloidal surrogate to the Poisson likelihood and is parallelizable and fairly simple to implement. We use ordered subsets and pre-computed surrogate curvatures to accelerate convergence and reduce computation. When a single subset is used with appropriate curvatures, the algorithm monotonically decreases the cost function. This is about the most that can be said about convergence since the cost function is not convex.

When compared with the post-processing technique of Joseph and Spital (1978), the statistical algorithm yielded fewer artefacts. The JS method estimates the line-integraldependent nonlinearity for each sinogram bin and then recalculates the line integrals. The statistical method needs no such post-processing since it inherently accounts for the nonlinearities. This is likely the reason for its higher accuracy. Displacement model 
reconstruction gives images with reduced artefacts in anatomical objects. The solution model is promising for quantitative estimation of mineral solution density and may have clinical utility in bone mineral density studies. Many structures within bone can be characterized as mineral solutions and quantitative estimates of their densities are useful in understanding the progress of certain diseases.

The displacement model was less accurate in estimating the density of fat and breast tissue. As seen in figure 1, fat spectral properties deviate most from water, when compared to other soft tissues. This deviation may explain the inaccuracy in estimating fat density. In the case of breast tissue, its density is very close to that of water and this contributes to the difficulty in visually distinguishing it from water in the reconstructed images. The linear attenuation coefficient of breast is also very close to that of water at clinical $\mathrm{kVp}$ settings. In applications where fat must be estimated accurately, a modified object model is necessary. One possibility, for example, is to use the mass attenuation coefficient of fat as a base substance instead of water. Another alternative would be to segment the fat regions of an image and model the beam hardening nonlinearity they introduce in the projection. It may also be possible to generalize the tissue fraction functions to include the known spectral properties of different tissues.

The solution model did not incorporate the nonlinear dependence of solution density on mineral concentration. This dependence can be expressed in terms of a low-order polynomial fit to empirical measurements of solution density and concentration. This polynomial fit can be used to post-correct the image, or can be incorporated in the design of the solution model fraction functions.

Future work will include applying the polyenergetic approach to objects with three or more tissue types. The bone/soft-tissue model is sufficient for most cases, but a three-class model is necessary when contrast agents such as iodine are introduced (Joseph and Ruth 1997) and possibly when metallic implants are present. A three-substance model may also help accurately estimate the density of fat.

Noise performance of statistical iterative algorithms depends on how close the assumed statistical model is to actual measurements. It has been shown that the ordinary Poisson model does not capture the full complexity of the measurement process in CT, and does not take into account the dependence of the measurement statistics on the beam spectrum. A more realistic model is the compound Poisson model (Elbakri and Fessler 2003, Whiting 2002). In future work we hope to present an algorithm based on a compound Poisson likelihood.

Future work will also address some of the challenges posed by the penalized-likelihood approach. Penalized likelihood is attractive because it improves the conditioning of the problem and enables one to choose penalty functions that control desired properties such as edge preservation. One drawback, however, is the absence of an intuitive method for choosing the values of the regularization parameters, which is often done by trial and error. This is inefficient and time consuming, and there is a need for a more systematic method for choosing the parameters. Another undesirable property of penalized-likelihood image reconstruction is its non-uniform spatial resolution (Fessler and Rogers 1996). A remedy for quadratic penalties exists (Stayman and Fessler 2000). For transmission imaging, we prefer to use the edge-preserving Huber penalty, for which the non-uniform resolution problem has not been addressed.

Recent advances with dual-energy statistical reconstruction (Fessler et al 2002, Sukovic and Clinthorne 2000) have offered another approach for polyenergetic image reconstruction. Dual energy provides an additional set of measurements that enable reconstruction of separate bone and water images. It will be interesting in the future to compare the performance of single-energy and dual-energy statistical reconstruction. 


\section{Acknowledgments}

This work was supported in part by NIH grants R01 CA60711 and 1P01 CA87634-01. The authors are grateful to N Clinthorne and P Sukovic for the data used in section 4, and to M Goodsitt for valuable discussions.

\section{References}

Alvarez R E and Macovski A 1976 Energy-selective reconstruction in x-ray computerized tomography Phys. Med. Biol. $21733-44$

Boone J M and Seibert J A 1997 An accurate method for computer-generating tungsten anode X-ray spectra from 30 to $140 \mathrm{kV}$ Med. Phys. 24 1661-70

Brooks R A and Chiro G D 1976 Beam hardening in X-ray reconstruction tomography Phys. Med. Biol. 21 390-8

Cardinal H N and Fenster A 1990 An accurate method for direct dual-energy calibration and decomposition Med. Phys. 17 327-41

Cody D D and Flynn M J 1989 A technique for measuring regional bone mineral density in human lumbar vertebral bodies Med. Phys. 16 766-72

De Man B, Nuyts J, Dupont P, Marchal G and Suetens P 2001 An iterative maximum-likelihood polychromatic algorithm for CT IEEE Trans. Med. Imaging 20 999-1008

De Pierro A R 1993 On the relation between the ISRA and the EM algorithm for positron emission tomography IEEE Trans. Med. Imaging 12 38-333

De Pierro A R 1995 A modified expectation maximization algorithm for penalized likelihood estimation in emission tomography IEEE Trans. Med. Imaging 14 132-7

Elbakri I A 2003 Statistical reconstruction algorithms for polyenergetic x-ray computed tomography PhD Thesis University of Michigan, Ann Arbor, MI

Elbakri I A and Fessler J A 2002 Statistical image reconstruction for polyenergetic x-ray computed tomography IEEE Trans. Med. Imaging 21 89-99

Elbakri I A and Fessler J A 2003 Efficient accurate likelihood for iterative image reconstruction in X-ray computed tomography Proc. SPIE 4032, Medical Imaging 2003: Image Proc.

Erdoğan H and Fessler J A 1999 Monotonic algorithms for transmission tomography IEEE Trans. Med. Imaging 18 801-14

Fessler J A, Elbakri I A, Sukovic P and Clinthorne N H 2002 Maximum-likelihood dual-energy tomographic image reconstruction Proc. SPIE 4684, Medical Imaging 2002: Image Proc.

Fessler J A and Rogers W L 1996 Spatial resolution properties of penalized-likelihood image reconstruction methods: space-invariant tomographs IEEE Trans. Imaging Proc. 5 1346-58

Glover G H 1982 Compton scatter effects in CT reconstructions Med. Phys. 9 860-7

Hsieh J, Molthen R C, Dawson C A and Johnson R H 2000 An iterative approach to the beam hardening correction in cone beam CT Med. Phys. 27 23-9

Hubbell J H and Seltzer S M 1997 Tables of X-ray Mass Attenuation Coefficients and Mass Energy-Absorption Coefficients http://physics.nist.gov/xaamdi

Joseph P M and Ruth C 1997 A method for simultaneous correction of spectrum hardening artefacts in CT images containing both bone and iodine Med. Phys. 24 1629-34

Joseph P M and Spital R D 1978 A method for correcting bone induced artefacts in computed tomography scanners J. Comput. Assisted Tomogr. 2 100-8

Joseph P M and Spital R D 1982 The effects of scatter in X-ray computed tomography Med. Phys. 9 464-72

Kak A C and Slaney Malcolm 1988 Principles of Computerized Tomographic Imaging (New York: IEEE Press)

Meagher J M, Mote C D and Skinner H B 1990 CT image correction for beam hardening using simulated projection data IEEE Trans. Nucl. Sci. 37 1520-4

Nalcioglu O and Lou R Y 1979 Post-reconstruction method for beam hardening in computerized tomography Phys. Med. Biol. 24 330-40

Rao G U, Yaghmai I, Wist A O and Arora G 1987 Systematic errors in bone-mineral measurements by quantitative computed tomography Med. Phys. 14 62-9

Ruth C and Joseph P M 1997 Estimation of a photon energy spectrum for a computed tomography scanner Med. Phys. 24 695-702

Snyder D L, Helstrom C W, Lanterman A D, Faisal M and White R L 1995 Compensation for read-out noise in CCD images J. Opt. Soc. Am. A 12 273-83

Stayman J W and Fessler J A 2000 Regularization for uniform spatial resolution properties in penalizedlikelihood image reconstruction IEEE Trans. Med. Imaging 19 601-15 
Sukovic P and Clinthorne N H 1999 Design of an experimental system for dual energy x-ray CT Proc. IEEE Nucl. Sci. Symp. Med. Imaging Conf. vol 2 pp 1021-2

Sukovic P and Clinthorne N H 2000 Penalized weighted least-squares image reconstruction in single and dual energy $\mathrm{X}$-ray computed tomography IEEE Trans. Med. Imaging 19 1075-81

Tsui B M W, Terry J A and Gullberg G T 1993 Evaluation of cardiac cone-beam SPECT using observer performance experiments and ROC analysis Invest. Radiol. 28 1101-12

Whiting B R 2002 Signal statistics in x-ray computed tomography Proc. SPIE 4682, Medical Imaging 2002 Med. Phys.

Williamson J F, Whiting B R, Benac J, Murphy R J, Blaine G J, O'Sullivan J A, Politte D G and Snyder D L 2002 Prospects for quantitative computed tomography imaging in the presence of foreign metal bodies using statistical image reconstruction Med. Phys. 29 2404-18

Woodard H Q and White D R 1986 The composition of body tissues Br. J. Radiol. 59 1209-18

Yan C H, Whalen R T, Beaupré G S, Yen S Y and Napel S 2000 Reconstruction algorithm for polychromatic CT imaging: application to beam hardening correction IEEE Trans. Med. Imaging 19 1-11 